\title{
Arborization of Aqueous Chlorides in Pulsed Electromagnetic Fields as a Justification of Their Ability to Initiate the Formation of New Neuronal Dendrites
}

\author{
Yuri Pivovarenko \\ Research and Training Centre 'Physical and Chemical Materials Science', Kyiv Taras Shevchenko University and NAS of Ukraine, Kiev, \\ Ukraine
}

\author{
Email address: \\ y.pivovarenko@gmail.com
}

\section{To cite this article:}

Yuri Pivovarenko. Arborization of Aqueous Chlorides in Pulsed Electromagnetic Fields as a Justification of Their Ability to Initiate the Formation of New Neuronal Dendrites. International Journal of Neurologic Physical Therapy. Vol. 5, No. 1, 2019, pp. 21-24.

doi: $10.11648 /$ j.ijnpt.20190501.14

Received: May 11, 2019; Accepted: June 10, 2019; Published: June 25, 2019

\begin{abstract}
It is well known that low-frequency pulsed electromagnetic fields are able to stimulate the restoration of damaged neural contacts. Despite the fact that this effect is widely used by physicians, the physical basis of such an action of lowfrequency pulsed electromagnetic fields remains unknown. In this aspect, it is of interest that the drying of chloride solutions prepared in negatively charged water is accompanied by the formation of tree-shaped crystals, i.e. their arborization. Based on this, it was suggested that low-frequency pulsed electromagnetic fields cause negative electrification of aqueous solutions of chlorides, which are the main inorganic anions of nerve tissues, and, as a result, axonal arborization of neurons. During the experimental verification of such an assumption, the formation of tree-like crystals in drying solutions of chlorides was detected, through which weak constant and low-frequency pulsed electric currents were previously passed. This made it possible to suggest an explanation of the nature of axonal arborization of neurons, including those damaged, which is observed under the influence of low-frequency pulsed electromagnetic fields in vivo. Since chlorides are the main inorganic anions of blood, it was also proposed to explain the formation of new capillaries under the action of low-frequency pulsed electromagnetic fields. After a more detailed analysis, it was suggested that this kind of negative electrization of aqueous solutions of chlorides was due to free hydrogen atoms, which are the products of water electrolysis. However, it was suggested that oxygen atoms, which also appear during the electrolysis of water, are bound by chloride anions to form hypochlorite anions. Thus, such oxygen atoms are not able to cause a positive electrification of aqueous media in which there are electrical currents, including currents, caused by low-frequency pulsed electromagnetic fields. Thus, the importance of chlorine anions for regenerative processes, in general, was justified. However, it has been suggested that these hypochlorite anions can stimulate cell proliferation, as well as other active forms of oxygen. Thus, an understandable physicochemical basis of the therapeutic effects of low-frequency pulsed electromagnetic fields was proposed and partially experimentally established.
\end{abstract}

Keywords: Arborization, Neuron, Regeneration, Axonal Arborization, Dendrite, Pulsed Electromagnetic Field, PEMF

\section{Introduction}

It is well known that low-frequency pulsed electromagnetic fields (PEMFs) are capable of regenerating neural tissues. It is also well known that low-frequency PEMFs can stimulate axonal arborization of damaged neurons and restore impaired neural contacts. However, the nature of such an action of PEMFs is still considered unknown [1-16].
To find out the physicochemical basis of the regenerative effects of low-frequency PEMFs on the nervous system, we studied their effect on the crystallization of chlorides, which are the main inorganic anions, both in the cytoplasm of neurons and in their environment. At the same time, we took into account the characteristic ability of chlorides to form tree-like crystals in environments with a negative electric potential [17-21].

The results obtained during this study are presented here. 


\section{Materials and Methods}

During the experiments, low-frequency $(0 \div 100 \mathrm{~Hz})$ PEMFs generators of various designs, including home-made, were used.

All salts were purchased from «Ukreachim» (Ukraine).

\section{Results}

\subsection{Arborization of Chlorides Under the Action of Low-Frequency PEMFs on Their Aqueous Solutions}

In the course of numerous experiments, it was found that drying of various aqueous solutions of chlorides, previously exposed to low-frequency PEMFs $(0-100 \mathrm{~Hz})$, is accompanied by the formation of tree-like crystals (Figure 1).

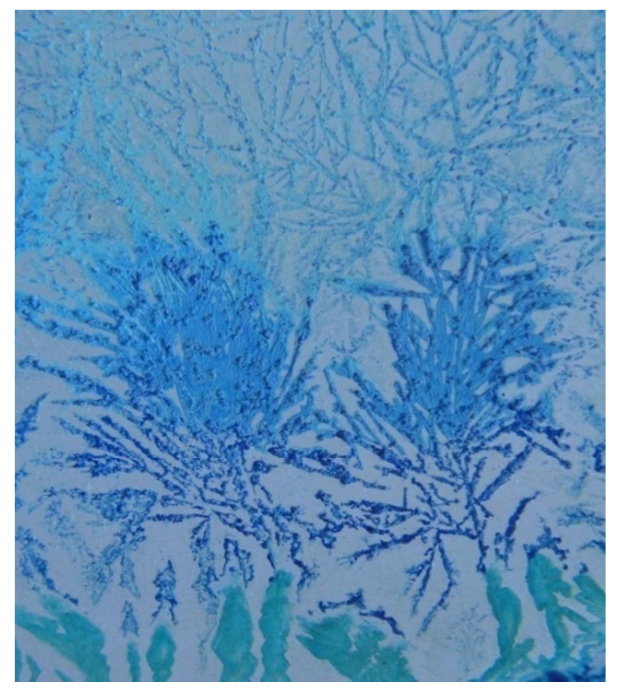

Figure 1. The crystals formed after drying an aqueous solution of $\mathrm{CuCl}_{2}$, which was previously subjected to the action of EMF, pulsing with a frequency of $10 \mathrm{~Hz}$ for 10 minutes. For contrast, the crystals formed were treated with ammonia vapors.

\subsection{Arborization of Chlorides, in Water Solutions of Which Weak Constant Currents Previously Flowed}

In the course of numerous experiments, it was found that tree-like crystals are also formed during the drying of various aqueous solutions of chlorides, through which short-term constant electric currents flowed (Figure 2).

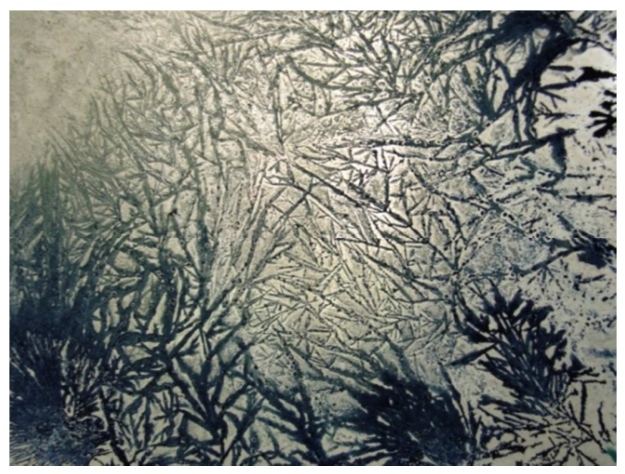

Figure 2. The crystals formed after drying an aqueous solution of $\mathrm{CuCl}_{2}$, through which a direct current of $10 \mathrm{~mA}$ was passed for 10 minutes.

\section{Discussion}

It was previously shown that the shape of crystals formed after evaporation of chloride solutions depends on the sign of the electric potential of water used to prepare such solutions. In particular, it was shown that the evaporation of chloride solutions prepared in water with a negative electric potential is accompanied by the formation of tree-like crystals (Figure 3) [17-21].

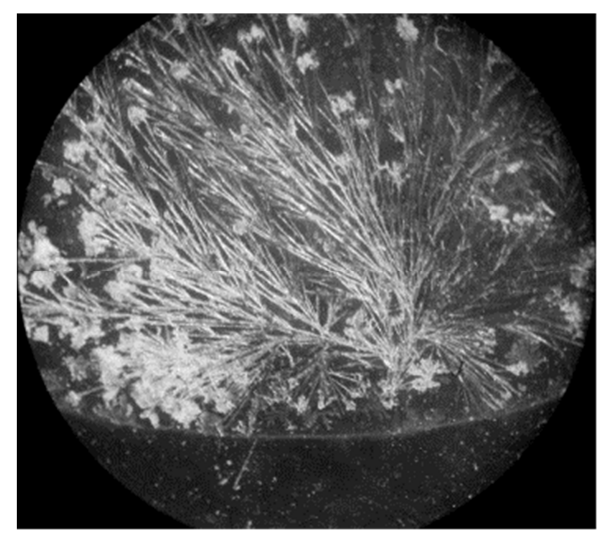

Figure 3. Crystals formed after drying of the $\mathrm{NaCl}$ solution prepared with water with the potential of $-200 \mathrm{mV}$ [17-21].

Based on this, it was concluded that low-frequency PEMFs cause negative electrification of aqueous solutions of chlorides. Considering that the tree crystals of chlorides are formed in solutions through which direct currents flowed (Figure 2), it was also concluded that the frequency of the PEMF used is not fundamentally important for negative electrification of water.

Taking all this into account, this explanation of the observed negative electrization of aqueous solutions of chlorides was proposed (Figures 1,2). When electric currents flow in aqueous solutions containing chloride anions, at least two chemical reactions occur [22]: $1 . \mathrm{H}_{2} \mathrm{O} \rightarrow \mathrm{H}_{2}+\mathrm{O}^{*}$ (the electrolysis of water); $2 . \mathrm{O}^{*}+\mathrm{Cl}^{-} \rightarrow \mathrm{ClO}^{-}$. Taking into account both of these reactions, it becomes clear why the concentration of hydrogen gas increases in aqueous solutions of chlorides through which various electrical currents flow. However, given that contact with hydrogen gas leads to negative electrification of water and aqueous solutions [22], it also becomes clear why aqueous solutions of chlorides through which electric currents flow acquire a negative charge.

Consequently, under the influence of low-frequency PEMFs, conditions can be created that facilitate the arborization of both the chlorine-rich cytoplasm of neurons and the neural environment, which is also rich in chlorides. Thus, under the influence of low-frequency PEMFs, conditions that are favorable for the formation of new dendrite outgrowths of neurons may well form in the nervous tissues.

But this is not all that can be explained with the help of the proposed reactions. So, one should take into account the formation of hypochlorite $\mathrm{ClO}^{-}$anions, which can also be 
formed under the influence of low-frequency PEMFs. This is important because the hypochlorite anion $\mathrm{ClO}^{-}$is one of the active forms of oxygen (ROS) [23], which stimulates cell proliferation $[24,25]$. It is also important to take into account that the hypochlorite ion $\mathrm{ClO}^{-}$can quickly decompose with the formation of atomic oxygen $\left(\mathrm{ClO}^{-} \rightarrow \mathrm{Cl}^{-}+\mathrm{O}^{*}[22]\right)$, which is capable of forming other ROS [23]. Given this, the stimulating effect of low-frequency PEMFs on the proliferation of nerve tissue cells can be clearly explained.

Thus, thanks to the results obtained and their understandable explanation, the described cases of restoration of the innervation of the affected tissues under the action of low-frequency PEMFs [1-16] received an acceptable, in my opinion, physico-chemical rationale.

It seems appropriate to make one important addition. Given that sodium chloride is the most common salt component of blood plasma, it can be assumed that the capillaries can also be updated under the influence of low-frequency PEMFs. Agree, this is important for the successful regeneration of neural tissues. In addition, it is also important for the successful regeneration of other tissues and organs. However, it should also be noted that the importance of chlorides for humans is usually ignored. Such inattention to chlorides causes, at a minimum, surprise, especially considering the exceptional role of chlorides in the formation of nerve impulses.

In conclusion, it can be added that the same reasons can cause bone regeneration, which, as is known [26-28], are also quickly restored under the action of the same lowfrequency PEMFs. In particular, it can be assumed that lowfrequency PEMFs can initiate the formation of polyphosphate fibers in newly formed bones. This assumption is supported by the ability of phosphates to form needle-like and threadlike crystals in negatively charged water [17-21].

\section{Conclusion}

Low-frequency PEMFs cause negative electrification of aqueous solutions of chlorides, as well as the arborization of the latter. Taking into account that chlorides are the most common anions in nerve tissues, their arborization can initiate axonal arborization of neurons, in general, which is observed under the action of low-frequency PEMFs. Thus, the chlorides of nerve tissues are necessary not only for the transmission of nerve impulses, but also for the restoration of damaged neurons, in particular, under the action of lowfrequency PEMFs.

The frequency of PEMFs used to regenerate the affected tissues may not be fundamentally important to achieve the desired healing effect.

\section{References}

[1] Raji A. M. (1984) An experimental study of the effects of pulsed electromagnetic field (Diapulse) on nerve repair. J. Hand Surg. [Br], 9 (2), 105-112.
[2] Sisken B. F., Kanje M., Lundborg G., Herbst E., Kurtz W. (1989) Stimulation of rat sciatic nerve regeneration with pulsed electromagnetic fields. Brain Res., 485 (2), 309316 .

[3] Bassett C. A. L. (1989) Fundamental and practical aspects of therapeutic uses of pulsed electromagnetic fields (PEMFs). Crit. Rev. Biomed. Eng., 17, 451-529.

[4] Kanje M., Rusovan A., Sisken B., Lundborg G. (1993) Pretreatment of rats with pulsed electromagnetic fields enhances regeneration of the sciatic nerve. Bioelectromagnetics, 14 (4), 353-359.

[5] Bassett C. A. (1993) Beneficial effects of electromagnetic fields. J. Cell Biochem., 51 (4), 387-393.

[6] Kanje M., Rusovan A., Sisken B., Lundborg G. (1993) Pretreatment of rats with pulsed electromagnetic fields enhances regeneration of the sciatic nerve. Bioelectromagnetics, 14 (4), 353-359.

[7] Liboff A. R., Smith S. D., McLeod B. R.(1995) Comment on "clarification and application of an ion parametric resonance model for magnetic field interactions with biological sysems". Bioelectromagnetism, 16, 272-273.

[8] Byers J. M., Clark K. F., Thompson G. C. (1998) Effect of pulsed electromagnetic stimulation on facial nerve regeneration. Arch Otolaryngol. Head Neck Surg., 124 (4), 383-389.

[9] Tyshkevich T. G., Nikitina V. V. (1998) Magnetic and electrical stimulation in the rehabilitative treatment of patients with organic lesions of the nervous system. Neurosci. Behav. Physiol., 28 (5), 594-597.

[10] Longo F. M., Yang T., Hamilton S., Hyde J. F., Walker J., Jennes L., Stach R., Sisken B. F. (1999) Electromagnetic fields influence NGF activity and levels following sciatic nerve transection. J Neurosci Res., 55 (2), 230-237.

[11] Goodwin T. J. (2003) Physiological and Molecular Genetic Effects of Time-Varying Electromagnetic Fields on Human Neuronal Cells; Technical Report of NASA Johnson Space Center; Houston, TX, United States.

[12] Crowe M. J., Sun Z. P., Battocletti J. H., Macias M. Y., Pintar F. A., Maiman D. J. (2003) Exposure to pulsed magnetic fields enhances motor recovery in cats after spinal cord injury. Spine, 28 (24), 2660-2666

[13] Baptista A. F,. Goes B. T., Menezes D., Gomes F. C., Zugaib J., Stipursky J., Gomes J. R., Oliveira J. T., Vannier-Santos M. A., Martinez A. M. (2009) PEMF fails to enhance nerve regeneration after sciatic nerve crush lesion. J. Periphery Nerv. Syst., 14 (4), 285-293.

[14] Kavlak E., Belge F., Unsal C., Uner A. G., Cavlak U., Cömlekçi S.(2014) Effects of pulsed electromagnetic field and swimming exercise on rats with experimental sciatic nerve injury. J. Phys. Ther. Sci., 26 (9), 1355-1361.

[15] Reardon S. (2016) Performance boost paves way for 'brain doping'. Nature, 531, 283-284.

[16] Minoo S., Payman T., Rahim M. (2017) Effects of Pulsed Electromagnetic Fields on Peripheral Nerve Regeneration Using Allografts in Sciatic Nerve: An Animal Model Study. Biomed J Sci \& Tech. Res., 1-7. 
[17] Pivovarenko Y. (2016) Nature of the polymorphism of salt crystals in the aspect of arborization diagnostic method. Morphologia, 10(1), 72-76.

[18] Pivovarenko Y. (2017) The Electric Potential of the Tissue Liquids of Living Organisms as a Possible Epigenetic Factor. Chemical and Biomolecular Engineering, 2 (3), 159-164.

[19] Pivovarenko Y. (2018) \pm Water: Demonstration of water properties, depending on its electrical potential. World Journal of Applied Physics, 3 (1), 13-18.

[20] Pivovarenko Y. (2018) Arborization of salt crystals in the aspect of plant morphology. Morphologia.;12 (1):37-41.

[21] Pivovarenko Y. (2018) The electric potential of the female body liquids and the effectiveness of cloning; Chapter 2 in: Top 10 Contributions on Biomedical Sciences. Hyderabad, India: Avid Science.

[22] Nekrasov B. V. (1974) Bases general chemistry, 1. Moscow: Chemistry. In Russian.

[23] Vladimirov Yu. A. (2004) Reactive forms of oxygen and nitrogen: significance for diagnosis, prophylaxis and therapy. Biochemistry, 69(1), 5-7. In Russian.
[24] Burdon R. H. (1995) Superoxide and hydrogen peroxide in relation to mammalian cell proliferation. Free. Radic. Biol. Med., 18, 775-794.

[25] Suzuki Y. J., Forman H. J., Sevanian A. (1997) Oxidants as stimulators of signal transduction. Free. Radic. Biol. Med., 22, 269-285.

[26] Ongaro A., Pellati A., Bagheri L., Fortini C., Setti S., De Mattei M. (2014) Pulsed electromagnetic fields stimulate osteogenic differentiation in human bone marrow and adipose tissue derived mesenchymal stem cells. Bioelectromagnetics, 35 (6), 426-436.

[27] Wang J., Tang N., Xiao Q., Zhang L., Li Y., Li J., Wang J., Zhao Z., Tan L. (2015) Pulsed electromagnetic field may accelerate in vitro endochondral ossification. Bioelectromagnetics, 36 (1), 35-44.

[28] Hannemann P. F., Mommers E. H., Schots J. P., Brink P. R., Poeze M. (2014) The effects of low-intensity pulsed ultrasound and pulsed electromagnetic fields bone growth stimulation in acute fractures: a systematic review and metaanalysis of randomized controlled trials. Arch Orthop Trauma Surg., 134 (8), 1093-106. 